\title{
Structure of weighted Hardy spaces on finitely connected domains
}

\author{
Nihat Gökhan Gögüş (iD \\ Sabanci University, Tuzla, 34956 Istanbul, Turkey
}

\begin{abstract}
We give a complete characterization of a certain class of Hardy type spaces on finitely connected planar domains. In particular, we provide a decomposition result and give a description of such functions through their boundary values. As an application, we describe an isomorphism from the weighted Hardy space onto the classical Hardy-Smirnov space. This allows us to identify the multiplier space of the mentioned Hardy type spaces as the space of bounded holomorphic functions on the domain.
\end{abstract}

Mathematics Subject Classification (2010). 30H10, 30J99

Keywords. Hardy space, decomposition, boundary values

\section{Introduction}

Recently, E. A. Poletsky and M. Stessin in [9] introduced a scale of Hardy and Bergman type spaces which consists of holomorphic functions on the underlying hyperconvex domain in the $n$-dimensional complex Euclidean space $\mathbb{C}^{n}$, where $n \geq 1$ is an integer. The class of hyperconvex domains contain a wide range of classical domains, in particular the $n$ dimensional unit ball and the polydisk in $\mathbb{C}^{n}$ are hyperconvex. This way, the theory of Hardy and Bergman spaces in the classical settings have been unified and generalized in [9]. Further studies in this direction will help us to construct a more unified system of methods to attack the problems of Hardy and Bergman spaces. Before the works of Poletsky and Stessin, M. A. Alan has already defined using a similar construction Hardy type spaces in hyperconvex domains in $\mathbb{C}^{n}$ in [1].

In this paper we concentrate on the $n=1$ case for the Hardy space construction of Poletsky and Stessin. By definition to each subharmonic function $u$ continuous near the boundary of $G$ corresponds a space, which is denoted by $H_{u}^{p}$ of holomorphic functions in $G$. Here $G$ is a bounded regular domain in $\mathbb{C}$. Throughout the paper these spaces will be called Poletsky-Stessin Hardy spaces. Following the motivating work of Poletsky and Stessin, the structure and first examples on the unit disk of Poletsky-Stessin Hardy spaces were further investigated in [2], [11] and [12].

Among these recent work, the author and M. A. Alan gave a complete characterization of $H_{u}^{p}$ spaces that live in the plane domains through the boundary values of the functions in this class or through a growth description of their harmonic majorants. As an application, a Beurling's type theorem was proved in [2]. This states roughly that if $G$ is the unit disk

Email address: nggogus@sabanciuniv.edu

Received: 29.07.2019; Accepted: 30.10.2019 
$\mathbb{D}$, and $u$ is such a subharmonic exhaustion on $\mathbb{D}$, there exists a bounded outer function $\varphi$ so that the space $H_{u}^{p}$ isometrically equals to $\mathcal{M}_{\varphi, p}, p>0$. Moreover, $\varphi$ belongs to the class $H_{u}^{p}$. Here we denote by $\mathcal{M}_{\varphi, p}$ the space $\varphi^{2 / p} H^{p}$ together with the norm defined by

$$
\|f\|_{\mathcal{M}_{\varphi, p}}:=\left\|f / \varphi^{2 / p}\right\|_{p}, \quad f \in \mathcal{M}_{\varphi, p}
$$

Using this result, we were able to construct several examples of holomorphic spaces with certain properties. Let us remark that the space $\mathcal{M}_{\varphi, 2}$ is a useful tool in the study of sub-Hardy Hilbert spaces (see [10]).

The problems and results addressed in this paper can be summarized as follows:

When $G$ is finitely connected:

(1) We provide a decomposition result of functions which belong to $H_{u}^{p}(G)$. (Theorem 4.1).

(2) We completely describe $H_{u}^{p}(G)$ in terms of their boundary values.

(3) We describe an isomorphism from the classical Hardy space $H^{p}(G)$ onto the space $H_{u}^{p}(G)$.

The last two results extend the research in [2], [11] and [12] from the disk to finitely connected planar domains. This paper is organized as follows: Section 2 is a brief summary of the previous work which are related and will be used in the paper. In section 3 we solve the inverse balayage problem for a given weight function. The main results of the paper are stated and proved in section 4 .

\section{Poletsky-Stessin-Hardy spaces}

In this section we will give the basic definitions to be used throughout the paper and recall some earlier results. Let $G \subset \mathbb{C}$ be a bounded domain. We say that a function $u \leq 0$ defined on $G$ is an exhaustion on $G$ if the level set

$$
B_{c, u}:=\{z \in G: u(z)<c\}
$$

is relatively compact in $G$ for any $c<0$. Let $u$ be an exhaustion and $c<0$, we set

$$
u_{c}:=\max \{u, c\}, \quad S_{c, u}:=\{z \in G: u(z)=c\} .
$$

Let $u \in \operatorname{sh}(G)$ be an exhaustion function. We assume that $u$ is continuous taking values in $\mathbb{R} \cup\{-\infty\}$ with the extended topology on this set. Following Demailly [4] we define

$$
\mu_{c, u}:=\Delta u_{c}-\chi_{G \backslash B_{c, u}} \Delta u .
$$

Here $\chi_{\omega}$ denotes the characteristic function for a given set $\omega \subset G$. We will denote the class of negative subharmonic exhaustion functions on $G$ by $\mathcal{E}(G)$. Also we denote by $\mathcal{E}_{0}(G)$ the class of all functions $u \in \mathcal{E}(G)$ such that $\int \Delta u<\infty$.

Following [9] we set

$$
s h_{u}(G):=s h_{u}:=\left\{v \in \operatorname{sh}(G): v \geq 0, \sup _{c<0} \int_{S_{c, u}} v d \mu_{c, u}<\infty\right\},
$$

and we define

$$
H_{u}^{p}(G):=H_{u}^{p}:=\left\{f \in \operatorname{hol}(G):|f|^{p} \in s h_{u}\right\}
$$

for $p>0$. Let us write

$$
\|v\|_{u}:=\sup _{c<0} \int_{S_{c, u}} v d \mu_{c, u}=\int_{G}(v \Delta u-u \Delta v)
$$

if $v$ is a nonnegative subharmonic function. We set

$$
\|f\|_{u, p}:=\sup _{c<0}\left(\int_{S_{c, u}}|f|^{p} d \mu_{c, u}\right)^{1 / p}
$$

if $f$ is a holomorphic function on $G$. We list relavant recollections of basic facts below: 
- $H_{u}^{p}$ is a Banach space when $p \geq 1$.

- The Demailly measure $\mu_{u}$ has finite mass if and only if the constant function (equivalently, all polynomials) $f \equiv 1$ belongs to $H_{u}^{p}$.

- Let $G$ be a bounded regular domain and let $w \in G$. The classical Green function $v(z)=g_{G}(z, w)$ is a subharmonic exhaustion for $G$.

- Let $G=\mathbb{D}$ be the unit disk and let $v(z)=\log |z|$. Then $H_{v}^{p}(\mathbb{D})$ is the usual Hardy space $H^{p}(\mathbb{D})$.

We denote by $P_{G}(z, w)$ the Poisson kernel for the domain $G$. The space of all holomorphic functions $f$ in $G$ so that $|f|^{p}$ admits a harmonic majorant in $G$ is denoted by $H^{p}(G)$ (see for example [5]). By [9], $H_{u}^{p} \subset H^{p}$. It follows by a well-known fact that when $G$ has a sufficiently smooth boundary, then a function $f$ from the space $H_{u}^{p}(G)$ possesses boundary values (almost everywhere non-tangential limits), which we denote by $f^{*}$. The usual normalized arclength measure on $\partial G$ is denoted by $\nu$. Hence, $\nu(\partial G)=1$.

Theorem 2.1 ([2, Theorem 2.10]). Suppose that $G$ is a Jordan domain with rectifiable boundary or a bounded domain with $C^{2}$ boundary. Let $p>1, f \in H^{p}(G)$ and let $u \in \mathcal{E}(G)$. Then the following statements are equivalent:

i. $f \in H_{u}^{p}(G)$.

ii. $f^{*} \in L^{p}\left(V_{u} \nu\right)$, where

$$
V_{u}(\zeta):=\int_{G} P_{G}(z, \zeta) \Delta u(z), \quad \zeta \in \partial G .
$$

iii. There exists a measure $\widetilde{\mu_{u}}$ on $\partial G$ so that $f^{*} \in L^{p}\left(\widetilde{\mu_{u}}\right)$. Moreover, if $E$ is any Borel subset of $\partial G$ with measure $\nu(E)=0$, then $\widetilde{\mu_{u}}(E)=0$ and we have the equality

$$
\int_{\partial G} \gamma d \widetilde{\mu_{u}}=\int_{G} P_{G}(\gamma) \Delta u
$$

for every $\gamma \in L^{1}(\nu)$.

In addition, if $f \in H_{u}^{p}(G)$, then $\|f\|_{u, p}=\left\|f^{*}\right\|_{L^{p}\left(\widetilde{\left.\mu_{u}\right)}\right.}$ and $d \widetilde{\mu_{u}}=V_{u} d \nu$.

Remark 2.2. i. If $G$ is the unit disk or a Jordan domain with rectifiable boundary, then the statements in Theorem 2.1 hold true for any $p>0$.

ii. Take a compact set $K \subset G$ so that $\Delta u(K)>r>0$. Let $m:=\min _{\zeta \in \partial G} \min _{z \in K} P_{G}(z, \zeta)$. Then $V_{u}(\zeta) \geq r m>0$ for every $\zeta \in \partial G$. Hence, replacing $u$ by $u /(r m)$, we may assume without loss of generality that $V_{u} \geq 1$ on $\partial G$.

iii. The weight function $V_{u}$ is lower semicontinuous. In fact, by Fatou's lemma

$$
\liminf _{j} V_{u}\left(\zeta_{j}\right)=\liminf _{j} \int_{G} P_{G}\left(z, \zeta_{j}\right) \Delta u(z) \geq \int_{G} P_{G}(z, \zeta) \Delta u(z)=V_{u}(\zeta) .
$$

Note that $V_{u}$ is the balayage of the measure $\Delta u$ on $\partial G$.

iv. Suppose $G$ is a bounded domain with $C^{2}$ boundary or a Jordan domain with rectifiable boundary. Let $u \in \mathcal{E}_{0}(G)$. Then

$$
u(z)=\int_{G} g_{G}(z, w) \Delta u(w), \quad z \in G .
$$

Since

$$
\frac{\partial g_{G}(\zeta, w)}{\partial n}=P_{G}(w, \zeta)
$$

when $\zeta \in \partial G$ and $w \in G, \frac{\partial u}{\partial n}(\zeta)$ exists for every $\zeta \in \partial G$. Here $\frac{\partial}{\partial n}$ denotes the normal derivative in the outward direction on $\partial G$ and

$$
\frac{\partial u(\zeta)}{\partial n}=V_{u}(\zeta)=\int_{G} P_{G}(w, \zeta) \Delta u(w), \quad \zeta \in \partial G
$$


Using the property (2.4) in Theorem 2.1 we see that

$$
\int_{\partial G} V_{u}(\zeta) d \nu(\zeta)=\int_{G} \Delta u=\int_{\partial G} \frac{\partial u}{\partial n}(\zeta) d \nu(\zeta) .
$$

Let $\varphi$ be a nonzero holomorphic function on the unit disk. Let $\mathcal{M}_{\varphi, p}$ denote the space $\varphi^{2 / p} H^{p}$ which we endow with the norm

$$
\|f\|_{\mathcal{M}_{\varphi, p}}:=\left\|f / \varphi^{2 / p}\right\|_{p}, \quad f \in \mathcal{M}_{\varphi, p}
$$

A function $\varphi \in H_{u}^{2}$ is called a $u$-inner function if $\left|\varphi^{*}(\zeta)\right|^{2} V_{u}(\zeta)=1$ for almost every $\zeta \in \partial \mathbb{D}$. If, moreover, $\varphi(z)$ is zero-free, we say that the function $\varphi$ is singular $u$-inner. The next result is proved in Theorem 3.2 and Corollary 3.3 in [2].

Theorem 2.3. Let $u \in \mathcal{E}(\mathbb{D})$. There exists a bounded $u$-inner and outer function $\varphi \in H_{u}^{2}$ so that $H_{u}^{2}=\mathcal{M}_{\varphi, 2}$. Moreover, these spaces are isometric.

The function $\varphi$ is uniquely determined (up to a unit constant). We have

$$
V_{u}\left(e^{i \theta}\right)=\frac{1}{\left|\varphi\left(e^{i \theta}\right)\right|^{2}}=\frac{1}{\varphi^{2}\left(e^{i \theta}\right)} \operatorname{sgn} \frac{1}{\varphi^{2}\left(e^{i \theta}\right)} .
$$

Here we set $\operatorname{sgn} \alpha:=|\alpha| / \alpha$ for any complex number $\alpha \neq 0$ and $\operatorname{sgn} 0:=0$.

Theorem 2.4. The set $L^{p}\left(V_{u} d \theta\right)$ coincides with $\varphi^{2 / p} L^{p}(d \theta)$ and the map $f \mapsto \varphi^{-2 / p} f$ is an isometric isomorphism from the space $L^{p}\left(V_{u} d \theta\right)$ onto $L^{p}(d \theta)$.

The next result reveals a complete factorization of functions belonging to the space $H_{u}^{p}(\mathbb{D})$.

Theorem 2.5 ([2, Theorem 3.4]). Let $0<p<\infty, f \in H_{u}^{p}(\mathbb{D}), f \not \equiv 0$, and $B$ be the Blaschke product formed with the zeros of $f$. There are zero-free $\varphi \in H_{u}^{2} \cap H^{\infty}, S \in H^{\infty}$ and $F \in H^{p}$ such that $\varphi$ is singular $u$-inner and outer, $S$ is singular inner, $F$ is outer, and

$$
f=B S \varphi^{2 / p} F .
$$

Moreover, $\|f\|_{p, u}=\|F\|_{p}$ and $H_{u}^{p}(\mathbb{D})=\mathcal{M}_{\varphi, p}$.

Rephrasing the last statement above we have a concrete isomorphism.

Corollary 2.6. The map $f \mapsto \varphi^{-2 / p} f$ is an isometric isomorphism from the space $H_{u}^{p}$ onto $H^{p}$

The following Lemma will be useful in the next section. Its proof is a simple calculation and we outline it here.

Lemma 2.7. Let $c$ be a number with $-1<c<0$. Then there exists a function $\kappa=\kappa_{c}$ defined on $(-\infty, 0]$ with the following properties:

i. $\kappa:(-\infty, 0] \rightarrow(-\infty, 0]$ is non-decreasing, convex and $C^{\infty}$,

ii. $\kappa$ is real-analytic in $(c, 0]$,

iii. $\kappa(t) \equiv c$ when $t \leq c, \kappa(0)=0$, and $\kappa^{\prime}(0)=1$.

Proof. Let $a:=-\frac{\ln (-c)}{e}, b:=\frac{-1}{\ln (-c)}$, and

$$
\kappa(t):=\left\{\begin{array}{cl}
c+e^{\frac{-a}{(t-c)^{b}},} & t>c, \\
c, & t \leq c .
\end{array}\right.
$$

Then

and

$$
\kappa^{\prime}(t)=\frac{1}{e(t-c)^{b+1}} e^{\frac{-a}{(t-c)^{b}}}
$$

$$
\kappa^{\prime \prime}(t)=\frac{1}{e(t-c)^{2 b+2}}\left(1 / e-(b+1)(t-c)^{b+1}\right) e^{\frac{-a}{(t-c)^{b}}}
$$


for $t>c$. For $t \leq c, \kappa^{\prime}(t)=\kappa^{\prime \prime}(t)=0$. It can be checked that $\kappa^{\prime \prime}(t)>0$ for $c<t \leq 0$, and $\kappa$ satisfies all properties in i., ii. and iii.

\section{Constructing subharmonic exhaustion from a given weight function}

Let $G$ be a Jordan domain with rectifiable boundary and $\psi$ be a given holomorphic function in $H^{1}(G)$. We consider in this section the problem of finding a subharmonic exhaustion $u$ on $G$ so that $V_{u}(\zeta)=|\psi(\zeta)|$ when $\zeta \in \partial G$. We can always suppose that $G=\mathbb{D}$ after a conformal map of $G$ onto $\mathbb{D}$. The following result was obtained for the disk in [6] (see also [8]).

Theorem 3.1. Let $G$ be a Jordan domain with rectifiable boundary, $\psi$ be a lower semicontinuous function on $\partial G$ so that $\psi \geq k$ for some constant $k>0$. Then there exists a function $u \in \mathcal{E}(G)$ so that $\psi=V_{u}$.

a. $u$ is the decreasing limit of functions in $\mathcal{E}_{0} \cap C^{\infty}(\bar{G})$ which converge uniformly to $u$ on $\bar{G}$.

b. $u \in \mathcal{E}_{0}(G)$ if and only if $\psi \in L^{1}(d \nu)$.

In this section we consider finitely connected domains. If $\Gamma$ is the rectifiable boundary of a Jordan domain, let $G^{*}$ and $G$ be the unbounded and bounded components of $\mathbb{C} \backslash \Gamma$, respectively. By a conformal map $\Phi$ the curve $\Gamma$ can be mapped onto the unit circle and $G^{*}$ can be mapped onto $\mathbb{D}^{*}$, the compliment in $\mathbb{C}$ of the closed unit disk. We say that a function $u^{*}$ defined on $G^{*}$ belongs to $\mathcal{E}\left(G^{*}\right)$ if the corresponding function $u:=u^{*} \circ \Phi^{-1}(1 / z)$ belongs to $\mathcal{E}(\mathbb{D})$. A holomorphic function $f$ on $G^{*}$ belongs to $H_{u^{*}}^{p}\left(G^{*}\right)$ if the function $f \circ \Phi^{-1}(1 / z)$ belongs to $H_{u}^{p}(\mathbb{D})$. Clearly $H_{u^{*}}^{p}\left(G^{*}\right) \subset H^{p}\left(G^{*}\right)$. We write $a \approx b$ if there exists an absolute constant $C>0$ so that $C^{-1} b \leq a \leq C b$.

Lemma 3.2. Let $\Gamma$ be the $C^{1+\varepsilon}$ boundary of a Jordan domain for some $\varepsilon>0$. Let $G^{*}$ be the unbounded component of $\mathbb{C} \backslash \Gamma$ and let $\psi$ be a lower semicontinuous function on $\Gamma$ so that $\psi \geq k$ for some constant $k>0$. Then there exists a function $v \in \mathcal{E}\left(G^{*}\right)$ so that $\frac{\partial v(\zeta)}{\partial n} \approx \psi(\zeta)$ on $\Gamma$.

Proof. Suppose first that $\Gamma$ is the unit circle. Let $u \in \mathcal{E}(\mathbb{D})$ be the function proved for $\psi$ in Theorem 3.1. The function $v(z)=u(1 / z)$ belongs to $\mathcal{E}\left(\mathbb{D}^{*}\right)$ and satisfies $\frac{\partial v(\zeta)}{\partial n}=\psi(\zeta)$ on $\Gamma$. If $\Gamma$ is a Jordan curve with $C^{1+\varepsilon}$ boundary and if $\Phi$ is a conformal map of $G$ onto $\mathbb{D}$, let $v(z):=u \circ \Phi(1 / z)$, where $u \in \mathcal{E}(\mathbb{D})$ is so that $\frac{\partial u(\zeta)}{\partial n}=\psi \circ \Phi^{-1}(1 / \zeta)$ for $|\zeta|=1$. By a classical result of Painlevé (cf. [7, Theorem 5.2.4]) $\Phi$ extends to a $C^{1}$ map on the closed set $\bar{G}$. Thus, the estimate holds.

Let $G$ be a bounded domain so that $\partial G=\cup_{j=0}^{N} \Gamma_{j}$, where each $\Gamma_{j}$ is a Jordan curve and $\Gamma_{j} \cap \Gamma_{k}=\emptyset$ when $j \neq k$. Let $G_{0}$ be the bounded component of $\mathbb{C} \backslash \Gamma_{0}$. We assume that each $\Gamma_{j}, j \neq 0$, is contained in $G_{0}$. Let $G_{j}^{*}$ be the domain with boundary $\Gamma_{j}$ which contains $G$. For simplicity, in the following theorem and in the rest of the paper we will assume that all $\Gamma_{j}$ are at least $C^{1+\varepsilon}$ for some $\varepsilon>0$, for all $j=0,1, \ldots, N$. The following theorem gives a full description of those weight functions produced by a subharmonic exhaustion (see Remark 2.2).

Theorem 3.3. Let $\psi$ be lower semicontinuous on $\partial G$ so that $\psi \geq s$ for some $s>0$. Then there exists a function $u \in \mathcal{E}(G)$ so that $\psi=V_{u} \approx \frac{\partial u}{\partial n}$. Moreover $u \in \mathcal{E}_{0}(G)$ if and only if $\psi \in L^{1}(\partial G, d \nu)$.

Proof. For any $j \in\{0,1, \cdots, N\}$ let $\psi_{j}(\zeta)=\psi(\zeta)$ if $\zeta \in \Gamma_{j}$. Let $v_{j} \in \mathcal{E}\left(G_{j}^{*}\right)$ be the function for $\psi_{j}$ given in Lemma 3.2 so that $V_{v_{j}}=\psi_{j}$ on $\partial G_{j}$. We can choose $c_{j}<0$ close to 0 so that the level set $B_{c_{j}, v_{j}}$ contains all $\Gamma_{k}$ for $k \neq j$. Let $c=\max \left\{c_{0}, \cdots, c_{N}\right\}$. Set $u_{j}(z):=\kappa_{c}\left(v_{j}(z)\right)$ and $u(z):=\sum_{j=0}^{N} u_{j}(z)-c N$ for $z \in G$. If $\zeta \in \Gamma_{j}$, then $u_{j}(\zeta)=0$ while 
$u_{k}(\zeta)=c$ when $k \neq j$. Hence $u(\zeta)=0$ and $u \in \mathcal{E}(G)$. On the other hand if $k \neq j$, then $u_{j}(z) \equiv c$ on an open set containing $\Gamma_{k}$, therefore by Lemma 3.2 and Lemma 2.7

$$
\frac{\partial u(\zeta)}{\partial n}=\frac{\partial u_{j}(\zeta)}{\partial n}=\frac{\partial v_{j}(\zeta)}{\partial n} \approx \psi(\zeta)
$$

when $\zeta \in \Gamma_{j}$. Together we have that $V_{u}=\psi$ on $\partial G$. The equivalence of $u \in \mathcal{E}_{0}$ and $\psi \in L^{1}$ follows directly from the identity

$$
\int_{\partial G} V_{u}(\zeta) d \nu(\zeta)=\int_{G} \Delta u
$$

\section{Decomposition results and the multiplier algebra of $H_{u}^{p}(G)$}

From the Cauchy integral formula if $f \in H(G)$, then $f$ has a unique decomposition of the form

$$
f(z)=f_{0}(z)+\ldots+f_{N}(z)
$$

where $f_{0} \in H\left(G_{0}\right), f_{j} \in H_{0}\left(G_{j}^{*}\right)$ if $j \in\{1, \ldots, N\}$, and $H_{0}\left(G_{j}^{*}\right)$ denotes the class of holomorphic functions in $H\left(G_{j}^{*}\right)$ that vanish at infinity. This holds true for bounded holomorphic functions; from [3] we see that

$$
H^{\infty}(G)=H^{\infty}\left(G_{0}\right)+H_{0}^{\infty}\left(G_{1}^{*}\right)+\ldots+H_{0}^{\infty}\left(G_{N}^{*}\right),
$$

where $H_{0}^{\infty}\left(G_{j}^{*}\right)=H^{\infty}\left(G_{j}^{*}\right) \cap H_{0}\left(G_{j}^{*}\right)$.

The next theorem tells us that if $G$ is finitely connected, the space $H_{u}^{p}(G)$ has a similar decomposition.

Theorem 4.1. Let $G$ be a finitely connected domain whose boundary consists of disjoint Jordan curves $\Gamma_{j}, 0 \leq j \leq N$, with rectifiable boundary so that $\Gamma_{0}$ surrounds $G$. Let $G_{0}$ be the bounded component of $\mathbb{C} \backslash \Gamma_{0}$ and for $j \in\{1, \ldots, N\}$, let $G_{j}^{*}$ be the domain with boundary $\Gamma_{j}$ which contains $G$. Let $u \in \mathcal{E}_{0}(G)$ and $p>0$. Then there exists $u_{0} \in \mathcal{E}_{0}\left(G_{0}\right)$, $u_{j} \in \mathcal{E}_{0}\left(G_{j}^{*}\right), j=1, \ldots, N$, so that every $f \in H_{u}^{p}(G)$ can be represented in the form

$$
f(z)=f_{0}(z)+\cdots+f_{N}(z),
$$

where $f_{0} \in H_{u_{0}}^{p}\left(G_{0}\right), f_{j} \in H_{u_{j}}^{p}\left(G_{j}^{*}\right) \cap H_{0}\left(G_{j}^{*}\right)$ for $j \in\{1, \ldots, N\}$.

Proof. Let $u \in \mathcal{E}_{0}(G)$ and $f \in H_{u}^{p}(G)$. Then the function $V_{u}$ is lower semicontinuous and $V_{u} \geq s$ on $\partial G$ for some $s>0$ (see Remark 2.2). By the proof of Theorem 3.3 it is deduced that there exists $\omega \in \mathcal{E}_{0}(G)$ so that $V_{\omega}=V_{u}$ and $\omega$ is of the form

$$
\omega=u_{0}+\cdots+u_{N}-c N,
$$

where $c<0, u_{j} \in \mathcal{E}_{0}\left(G_{j}^{*}\right)$ and $V_{u_{j}}=\left.V_{u}\right|_{\Gamma_{j}}$ for $0 \leq j \leq N$.

Since $H_{u}^{p}(G) \subset H^{p}(G)$ it is well-known that (see for example [5, Theorem 10.12]) $f(z)=$ $f_{0}(z)+\cdots+f_{N}(z)$, where $f_{j} \in H^{p}\left(G_{j}^{*}\right)$. In fact, each $f_{j}$ is of the form (after analytic continuation)

$$
f_{j}(z)=\int_{\Gamma_{j}} \frac{f(\zeta)}{\zeta-z} d \nu(\zeta)
$$

Hence for each $j$ the functions $f_{0}, f_{1}, \cdots, \hat{f}_{j}, \cdots, f_{N}$ are bounded on $\Gamma_{j}$. Here $\hat{f}_{j}$ means that $f_{j}$ is not in the list. Therefore, $\left|f_{j}(\zeta)\right|^{p} \leq|f(\zeta)|^{p}+m$ when $\zeta \in \Gamma_{j}$ for some constant $m$. The norm of $f_{j}$ is estimated as

$$
\begin{aligned}
\left\|f_{j}\right\|_{H_{u_{j}}^{p}\left(G_{j}^{*}\right)}^{p} & =\int_{\Gamma_{j}}\left|f_{j}(\zeta)\right|^{p} V_{u}(\zeta) d \nu \leq \int_{\Gamma_{j}}|f(\zeta)|^{p} V_{u}(\zeta) d \nu+m\left\|V_{u}\right\|_{L^{1}} \\
& \leq\|f\|_{H_{u}^{p}(G)}^{p}+m\left\|V_{u}\right\|_{L^{1}} .
\end{aligned}
$$


Since $f \in H_{u}^{p}(G)$ and $u \in \mathcal{E}_{0}(G),\|f\|_{H_{u}^{p}(G)}<\infty$ and $\left\|V_{u}\right\|_{L^{1}}<\infty$, hence $\left\|f_{j}\right\|_{H_{u_{j}}^{p}\left(G_{j}^{*}\right)}<\infty$ and $f_{j} \in H_{u_{j}}^{p}\left(G_{j}^{*}\right)$.

Finally, we describe the boundary values of functions from $H_{u}^{p}(G)$ and describe an isomorphism from $H_{u}^{p}(G)$ onto $H^{p}(G)$. We use the same assumptions and notations of Theorem 4.1 in the next statement.

Theorem 4.2. Let $u \in \mathcal{E}_{0}(G)$ and $p>0$. Then there exists $u_{0} \in \mathcal{E}_{0}\left(G_{0}\right), u_{j} \in \mathcal{E}_{0}\left(G_{j}^{*}\right)$, and zero-free functions $\varphi_{0} \in H^{\infty}\left(G_{0}\right) \cap H_{u_{0}}^{2}\left(G_{0}\right)$ and $\varphi_{j} \in H_{0}^{\infty}\left(G_{0}\right) \cap H_{u_{j}}^{2}\left(G_{j}^{*}\right), j=1, \ldots, N$, so that

i. $H_{u}^{p}(G)=\varphi_{0}^{2 / p} H^{p}\left(G_{0}\right)+\varphi_{1}^{2 / p} H^{p}\left(G_{1}^{*}\right)+\ldots+\varphi_{N}^{2 / p} H^{p}\left(G_{N}^{*}\right)$.

ii. If $f \in H_{u}^{p}(G)$, then $\left.f^{*}\right|_{\Gamma_{j}} \in L^{p}\left(V_{u_{j}} d \nu\right)$ for $j=0,1, \ldots, N$.

iii. $\|f\|_{H_{u}^{p}(G)} \approx\left\|f_{0}\right\|_{H_{u_{0}}^{p}\left(G_{0}\right)}+\sum_{j=1}^{N}\left\|f_{j}\right\|_{H_{u_{j}}^{p}\left(G_{j}^{*}\right)}$ for $f \in H_{u}^{p}(G)$, where $f(z)=f_{0}(z)+$ $\cdots+f_{N}(z)$ denotes the unique decomposition in (4.2).

iv. The mapping $T: H_{u}^{p}(G) \rightarrow H^{p}(G)$ given by

$$
T f=\sum_{j=0}^{N} f_{j} / \varphi_{j}^{2 / p}
$$

is a linear isomorphism of $H_{u}^{p}(G)$ onto $H^{p}(G)$.

Proof. Statements $i$. and ii. are direct consequences of Theorem 2.1, Theorem 2.5 and the discussions in this section. For the third statement, let $X$ denote the vector space $H_{u}^{p}(G)$ endowed with the norm

$$
\|f\|_{X}=\left\|f_{0}\right\|_{H_{u_{0}}^{p}\left(G_{0}\right)}+\sum_{j=1}^{N}\left\|f_{j}\right\|_{H_{u_{j}}^{p}\left(G_{j}^{*}\right)} .
$$

For $p \geq 1$, both $H_{u}^{p}(G)$ (with the usual norm) and $X$ are Banach spaces. For $0<p<1$, we don't have normed spaces, however, these are complete metric spaces. In fact, $H_{u}^{p}(G)$ and $X$ are closed subspaces of $L^{p}\left(V_{u} d \nu\right)$ and several consequences of Baire category theorem, such as closed graph theorem, carry over to both of these spaces. Notice that as sets both $H_{u}^{p}(G)$ and $X$ are the same. By closed graph theorem, we deduce that there exists a constant $C>0$ such that

$$
1 / C\|f\|_{X} \leq\|f\|_{H_{u}^{p}(G)} \leq C\|f\|_{X}
$$

for every $f \in H_{u}^{p}(G)$. This proves $i i i$.

By Corollary 2.6, after a conformal mapping of $G_{j}^{*}$ onto $\mathbb{D}$, any function $g \in H^{p}\left(G_{j}^{*}\right)$ is of the form $g=\varphi_{j}^{2 / p} h$ for some $h \in H^{p}\left(G_{j}^{*}\right)$ and $\|g\|_{H^{p}\left(G_{j}^{*}\right)} \approx\|h\|_{H^{p}\left(G_{j}^{*}\right)}$. It follows from statements $i$. and iii. that $T$ gives an isomorphism of $H_{u}^{p}(G)$ onto $H^{p}(G)$. This fisinishes the proof.

Let $X$ be a Banach space of holomorphic functions on $G$. The multiplier algebra $M(X)$ of $X$ is defined to be the class of holomorphic functions $g$ on $G$ such that $g f \in X$ for every $f \in X$. By the closed graph theorem a function $g \in H(G)$ belongs to the multiplier algebra $M\left(H_{u}^{p}(G)\right)$ if and only if the multiplication operator $M_{g}$ which assigns to a function $f \in H(G)$ the product $g f$ is a bounded operator on $H_{u}^{p}(G)$.

Corollary 4.3. For $p>0, M\left(H_{u}^{p}(G)\right)=H^{\infty}(G)$.

Proof. It is well-known that $M\left(H^{p}(\mathbb{D})\right)=H^{\infty}(\mathbb{D})$. Hence, if $\Gamma$ is the rectifiable boundary of a Jordan domain and if $U$ is a connected component (bounded or unbounded) of $\Gamma$, then $M\left(H^{p}(U)\right)=H^{\infty}(U)$. This can be seen by considering a conformal mapping from 
$\mathbb{D}$ onto $U$. By Theorem $4.2(i), H_{u}^{p}(G)=\varphi_{0}^{2 / p} H^{p}\left(G_{0}\right)+\varphi_{1}^{2 / p} H^{p}\left(G_{1}^{*}\right)+\ldots+\varphi_{N}^{2 / p} H^{p}\left(G_{N}^{*}\right)$. Let $g \in M\left(H_{u}^{p}(G)\right)$. Then from Theorem $4.2($ iii $)$, if $f \in H_{u}^{p}(G)$, then

$$
\|g f\|_{H_{u}^{p}(G)} \approx\left\|g f_{0}\right\|_{H_{u_{0}}^{p}\left(G_{0}\right)}+\sum_{j=1}^{N}\left\|g f_{j}\right\|_{H_{u_{j}}^{p}\left(G_{j}^{*}\right)}<\infty
$$

where $f_{j}$ 's are as in (4.2). Let $g(z)=\sum_{j=0}^{N} g_{j}(z)$ be the unique decomposition of $g$ as described in (4.1). Fix $j \in\{0, \ldots, N\}$. If $i \neq j$, then $g_{i}$ is bounded near $\Gamma_{j}$, therefore, $\left\|g_{i} f_{j}\right\|_{H_{u_{j}}^{p}\left(G_{j}^{*}\right)}<\infty$. Combining with (4.3), this means that $\left\|g_{j} f_{j}\right\|_{H_{u_{j}}^{p}\left(G_{j}^{*}\right)}<\infty$. Since $g_{j} \in H\left(G_{j}^{*}\right)$ and $f_{j}$ can be an arbitrary element of $H_{u_{j}}^{p}\left(G_{j}^{*}\right)$, we see that $g_{j} \in M\left(H_{u_{j}}^{p}\left(G_{j}^{*}\right)\right)$, therefore it is bounded on $G_{j}^{*}$. Hence, $g \in H^{\infty}(G)$ and we have proved that $M\left(H_{u}^{p}(G)\right) \subset$ $H^{\infty}(G)$. The reverse inclusion clearly holds from Theorem 4.2(iii). The proof is finished.

Acknowledgment. This work was supported by a TUBITAK grant project 118F405.

\section{References}

[1] M.A. Alan, Hardy spaces on hyperconvex domains, M.Sc. Thesis, Middle East Technical University, Ankara, 2003.

[2] M.A. Alan and N.G. Göğüs, Poletsky-Stessin-Hardy spaces in the plane, Complex Anal. Oper. Theory, 8 (5), 975-990, 2014.

[3] B. Chevreau, C.M. Pearcy and A.L. Shields, Finitely connected domains G, representations of $H^{\infty}(G)$, and invariant subspaces, J. Operator Theory, 6, 375-405, 1981.

[4] J.P. Demailly, Mesure de Monge Ampère et mesures plurisousharmonique, Math. Z. 194, 519-564, 1987.

[5] P.L. Duren, Theory of $H^{p}$ spaces, Pure and Applied Mathematics 38, Academic Press, New York-London, 1970.

[6] N. G. Göğüs, Structure of weighhted Hardy spaces in the plane, Filomat, 30 (2), 473-482, 2016.

[7] S. Krantz, Geometric function theory. Explorations in complex analysis, Cornerstones, Birkhäuser Boston, Inc., Boston, MA, 2006.

[8] E.A. Poletsky and K. Shrestha, On weighted Hardy spaces on the unit disk, Constructive approximation of functions, 195-204, Banach Center Publ. 107, Polish Acad. Sci. Inst. Math., Warsaw, 2015.

[9] E.A. Poletsky and M.I. Stessin, Hardy and Bergman spaces on hyperconvex domains and their composition operators, Indiana Univ. Math. J. 57 (5), 2153-2201, 2008.

[10] D. Sarason, Sub-Hardy Hilbert spaces in the unit disk, University of Arkansas Lecture Notes in the Mathematical Sciences, 10, John Wiley and Sons, Inc., New York, 1994.

[11] K.R. Shrestha, Weighted Hardy spaces on the unit disk, Complex Anal. Oper. Theory, 9 (6), 1377-1389, 2015.

[12] S. Şahin, Poletsky-Stessin Hardy spaces on domains bounded by an analytic Jordan curve in $\mathbb{C}$, Complex Var. Elliptic Equ. 60 (8), 1114-1132, 2015. 